\title{
Bayesian Network for Uncertainty Representation in Semantic Web: A Survey
}

\author{
Kumar Ravi \\ Department of Computer Applications, \\ S. Sinha College, Magadh University, \\ Aurangabad, Bihar, India
}

\author{
Sheopujan Singh \\ Department of Mathematics and Computer \\ Applications, \\ S. Sinha College, Magadh University, \\ Aurangabad, Bihar, India
}

\begin{abstract}
Bayesian network is a probabilistic model to represent uncertainty available in knowledge base and using it tremendous works have been done to prove its relevance in uncertainty representation and reasoning using Bayesian inference. Probability can be used to represent uncertainty like prediction information, situational awareness, data and knowledge fusion etc in knowledge base to implement various real life situations. Various approaches based on description logic, object oriented, entity relational, and first order logic have been tried to represent uncertainty successfully. One of them is Multi-Entity Bayesian Network (MEBN) logic to represent probabilistic information and performing knowledge fusion in ontology, which is realized using PR-OWL (Probabilistic Web Ontology Language). This paper aims at giving an overall view, the work carried out so far to represent uncertainty with the help of Bayesian Network in semantic web and a list of works done using MEBN/PR-OWL for knowledge fusion or the representation of uncertainty in semantic web.
\end{abstract}

Keywords: Bayesian Network, Uncertainty, PR-OWL, MEBN, Semantic Web

\section{INTRODUCTION}

Bayesian network is widely used method for the representation of uncertain data and knowledge [1]. Bayesian network is also known as recursive graphical models, Bayesian belief networks, belief networks, causal probabilistic networks, causal networks, influence diagram and many more. Bayesian network can be represented as Influence diagram by augmenting it with utility nodes and decision nodes, where utility node represents the value of a particular event and decision node represents the choices that might be made. Bayesian network has been used in various fields like medicine, forecasting, control, and modeling for human understanding to infer the knowledge using Bayesian Inference [20].

Bayesian inference is the most appealing technique to perform reasoning with Bayesian network, and learning Bayesian network parameters and structures to adapt the changes in the source of information, which can be performed using various methods like variable elimination and arc reversal, conditioning to perform inference in multiply-connected networks, logic sampling, Markov-Chain Monte Carlo methods etc [20].

Different representation techniques have been proposed to represent uncertain data and different uncertain reasoning algorithms have been implemented to get exact answer to the query and to retrieve relevant information from unorganized knowledge facts [16], [17], [19].

Uncertain reasoning employs various types of methodologies to deal with uncertain data to retrieve relevant and unambiguous information from knowledge base, where knowledge base can be represented in various forms according to type of uncertainty and its representation. Major components of knowledge base are concepts, roles, individuals and axioms, where concepts refer facts, roles are relationships between concepts, individuals are instances of concepts which follow properties of concepts, and axioms are relationships among concepts and roles.

Bayesian model and fuzzy logic model are two mathematical models to deal with various types of uncertainty viz. inconsistency, ambiguity, empirical, vagueness, incompleteness and inaccuracy (figure 1) [3]; as reported by Uncertainty Reasoning for the World Wide Web Incubator Group (URW3-XG), where Bayesian model is based on probability theory and fuzzy model is based on fuzzy logic. Probability theory can be used to represent and reason about inconsistency and ambiguity type of uncertainty in knowledge base. Fuzzy model can represent vagueness or fuzziness of facts in knowledge base.

Bayesian model is categorized into Bayesian network and Probabilistic extensions to description logic. Fuzzy logic model is categorized into first order probabilistic approach, fuzzy propositional logic and fuzzy description logic as shown in Figure 2. This paper concentrates mainly on Bayesian model, and available approaches based on Bayesian network for uncertainty representation and reasoning.

Bayesian network is the main part of Bayesian model, which is based on Bayes Theorem and its Product rule, which is main logic behind Bayesian inference. It uses joint probability distribution to represent each node along with its dependency on its predecessors in Directed Acyclic Graph (DAG), where DAG represents the degree of belief of events in the network. Each node will annotate with quantitative probability information i.e. conditional probability table. 


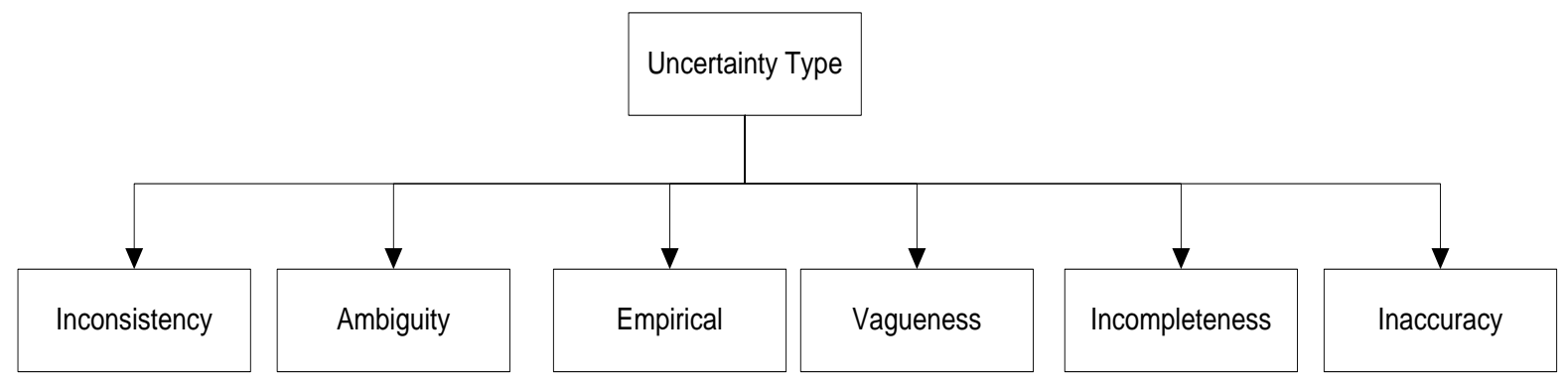

Figure 1. Uncertainty Type [3]

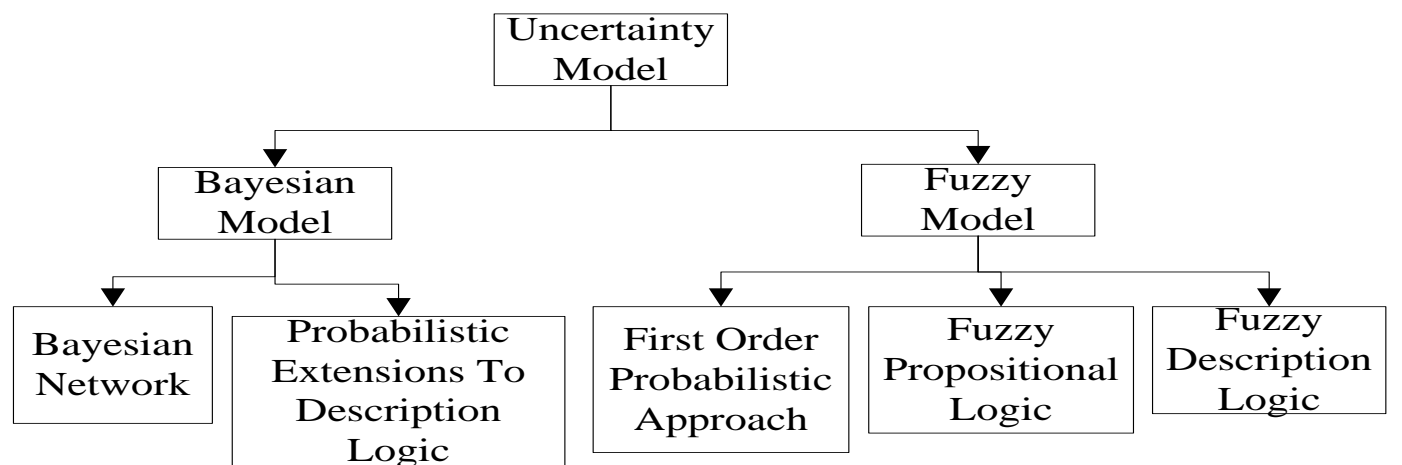

Figure 2. Uncertainty Model [3]

This paper provides some of the major existing representation and reasoning approaches regarding uncertainty representation using Bayesian network. The listed works in this paper can be classified into following two categories

a) Description Logic, Object Oriented and Entity Relation based Model

- P-Classic [22]

- $\quad$ SPOOK [23]

- Probabilistic Relational Model [30]

- DAPER Model [31]

b) Bayesian network with semantic web languages

- Probabilistic extension of RDF [4]

- $\quad$ Extension to $\mathrm{RDF}(\mathrm{S})$ [12]

- OntoBayes [25]

- BayesOWL [5]

- $\quad$ PR-OWL 2.0 [9], [11] and[18]

This paper is organized as follows: the paper gives semantics of Bayesian network with an example to make this paper selfcontained in the second section. Section 3 presents methodologies based on description logic, object-oriented, and entity relationship. Proposed works based on augmentation of Bayesian network with semantic languages viz. $\mathrm{RDF}, \mathrm{RDF}(\mathrm{S})$ and $\mathrm{OWL}$ are briefly discussed in section
4. A list of works done using PR-OWL and MEBN has been presented in section 5 and conclusion is given in section 6 .

\section{SEMANTICS OF BAYESIAN NETWORK}

Semantics of Bayesian Network (BN) can be viewed in two ways: the first one is the network as representation of joint probability distribution and second one is the encoding of a set of conditional independence statements [1]. The first view as; Bayesian network is a concise specification of any joint probability distribution, where a joint probability distribution will be able to answer a query or calculate the probability of an unknown event with given events. An uncertain attribute, feature, or hypothesis will be represented by random variable as a node in BN. The uncertainty of the dependence is shown as an edge between two nodes in DAG and represented as $\mathrm{P}\left(\mathrm{X}_{\mathrm{i}} \mid\right.$ parents $\left._{\mathrm{i}}\right)$ in conditional probability table, where $\mathrm{X}_{\mathrm{i}}$ is a node and parentsi is the parent node set of $\mathrm{X}_{\mathrm{i}}$. Each entry in the full joint probability distribution for a node will be calculated by taking values of affecting parent nodes in the form of conjunction of probability of each variable, such as $\mathrm{P}\left(\mathrm{X}_{1}=\mathrm{x}_{1} \wedge \ldots \wedge \mathrm{X}_{\mathrm{n}}=\mathrm{x}_{\mathrm{n}}\right)$ abbreviated as $\mathrm{P}\left(\mathrm{x}_{1}, \ldots, \mathrm{x}_{\mathrm{n}}\right)$.

$\mathrm{P}\left(\mathrm{x}_{1}, \ldots, \mathrm{x}_{\mathrm{n}}\right)=\prod_{i=1}^{n} P\left(x_{i} \mid\right.$ parents $\left.\left(x_{i}\right)\right)$ 


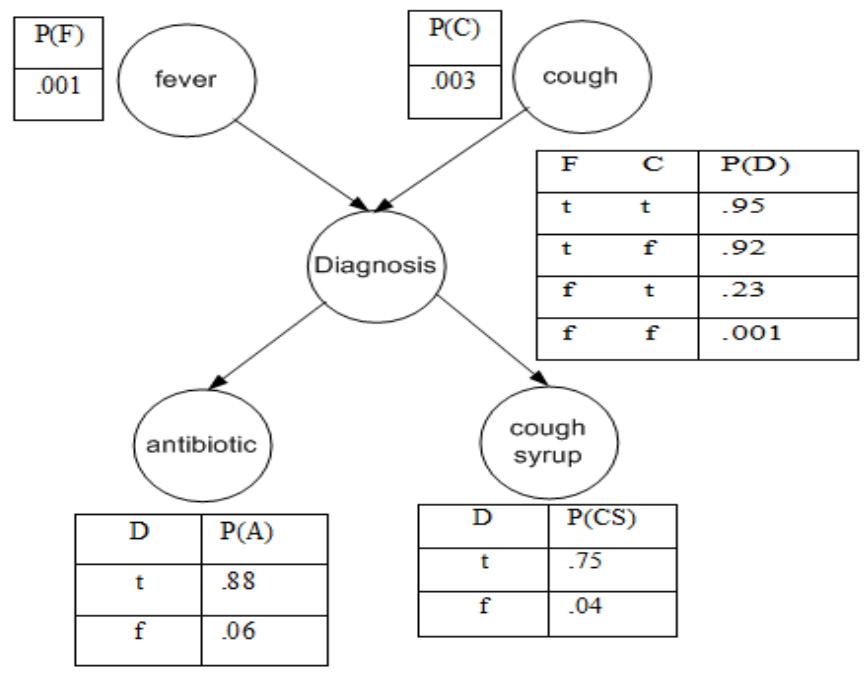

Figure 3 Bayesian Network for Medical Diagnosis

Figure 3 presents an example of Bayesian network, in which, a doctor has to diagnosis a person for fever or cough or both. Doctor can prescribe either antibiotic only or cough syrup only or both according to diagnosis. The probability of correct diagnosis, where person has neither fever nor cough where antibiotic and cough syrup both have been prescribed; will be calculated a-s follows

$$
\begin{aligned}
& \mathrm{P}(\mathrm{A} \wedge \mathrm{CS} \wedge \mathrm{D} \wedge \mathrm{F} \wedge \neg \mathrm{F} \wedge \neg \mathrm{C}) \\
& =\mathrm{P}(\mathrm{A} \mid \mathrm{D}) \mathrm{P}(\mathrm{CS} \mid \mathrm{D}) \mathrm{P}(\mathrm{D} \mid \neg \mathrm{F} \wedge \neg \mathrm{C}) \mathrm{P}(\neg \mathrm{F}) \mathrm{P}(\neg \mathrm{C}) \\
& =0.88 \times 0.75 \times 0.001 \times 0.999 \times 0.997 \\
& =0.00065736198
\end{aligned}
$$

\section{DESCRIPTION LOGIC, OBJECT ORIENTED AND ENTITY RELATION BASED MODEL}

Description Logic (DL) [10] can be viewed as stable representation of knowledge base for last decade. To represent uncertain information in description logic some researchers have been used Bayesian network, which can be seen as a part of probabilistic extensions to description logic.

Description logic is a subset of first order logic (FOL) to create a knowledge base. It uses terminological box (T- Box) to create vocabulary of concepts and assertions box (A-Box) to represent assertions about instances of concepts of T-Box or not, which makes easy to reason about knowledge base. It can be represented using resource description framework (RDF), resource description framework schema (RDFS) and web ontology language (OWL). OWL is available in 3 levels: OWL Lite, OWL DL and OWL Full. SHIF (D) is logic behind OWL Lite and SHOIN (D) for OWL DL, where OWL Lite has less expressivity than OWL DL and OWL DL has less expressivity than OWL Full.

\subsection{P-CLASSIC}

It is one of the first probabilistic versions of the DL Classic [22]. It supports terminological knowledge and uncertainty about properties of individual, the number of fillers for its roles, and the properties of these fillers is represented using Bayesian network. It has proposed inference procedure for probabilistic subsumption: computing the probability that a random individual in class $\mathrm{C}$ is also in class D. Knowledge representation formalisms can be based on a) rule-based languages b) object-centered formalism. This approach has integrated probabilities with object-centered language, which allows the specification of a probability distribution over the properties of individuals. Random variables of Bayesian network are the basic properties of individuals, the number of their fillers, and properties of their fillers. Probabilistic subsumption is provided to check the probability of belonging of complex concept $\mathrm{C}$ within the set of individuals in D. It gives better degree of overlap between two concepts, which was limited in CLASSIC description logic. It uses p-classes (probabilistic classes) to represent probabilistic component, each of which is a Bayesian network. A set of p-classes will represent probabilistic information of basic properties of individuals and other p-classes for role fillers.

CLASSIC uses two types of statement in its T-Box i.e. concept introductions and concept definitions, but terminology in P-CLASSIC will use only concept definitions and concept introductions are embedded with probabilistic component.

The major drawback of P-CLASSIC is unable to express equality relationship. Since different fillers are disjoint, and the number of fillers for each role is bounded. Some improvements were needed like support of disjunctive concepts, existential quantification, negation on arbitrary concepts (not only primitive ones) and qualified number restrictions. It doesn't include same-as constructor of CLASSIC DL.

\subsection{SPOOK: A system for probabilistic object-oriented knowledge representation}

This model is compact, modular, natural, and easy to build [23]. It is a unified model, which supports reusability and encapsulation. It can be used to develop and manage ontology of a large complex domain. It can also implement complex 
structured domain on the basis of objects and classes where uncertainty is represented using probability.

This is tested on military situation assessment, which have a large number of objects and those are related to one another in various ways. It has four major features like multicenteredness (i.e. each object can be accessed by a multitude of other objects, in a variety of ways), encapsulation, multivalued attribute and quantifier attribute, and structural uncertainty. Structural uncertainty is of two type i.e. number uncertainty and reference uncertainty. Number uncertainty implies uncertainty over the number of values of a multivalued complex attribute and reference uncertainty implies which is uncertainty over the value of a single valued complex attribute.

To perform query, it performs knowledge-based model construction at first and then Bayesian inference algorithm will be used. In the representation of various relationships among objects, it can violate the part-whole property and it cannot treat type uncertainty.

\subsection{Probabilistic Relational Model}

Probabilistic relational model (PRM) is a structured statistical model, which describes the domain using relational schema augmented with probabilistic distribution that is known as relational logic [30]. Frame-based logical representation is augmented with Bayesian network. Syntax is inspired from frame-based and object-oriented system so, it extends Bayesian network with objects, attributes and relationship.

PRM has three components: relational schema, probabilistic graphical model, and relational skeleton. A relational schema is a logical description of the domain of discourse, which will be transformed into a frame-based representation. It will use the concepts like relation for a class, column for an attribute of a class, and reference slot (opposite is inverse slot) for foreign key. A probabilistic graphical model is depicted using directed acyclic graph and can be represented in a logical formalism. A relational skeleton is specified for each class of a relational schema which will have a set of uninitialized objects.

Random variable will be defined for each uncertain attribute of the object where attributes of a same class or different class may be dependent on one another. To ensure acyclic representation of graph, it uses mainly two graphs: instance dependence graph and class dependence graph.

PRM provides various types of uncertainty like structural uncertainty, attribute uncertainty and class uncertainty, where structural uncertainty is in two forms: reference uncertainty and existence uncertainty. It uses two inference techniques for reasoning and learning purposes viz. exact inference and approximate inference.

\subsection{DAPER model}

The directed acyclic probabilistic entity-relationship model is a combination of ER-model with directed acyclic graph (DAG) and local distribution classes [31]. ER-model gives pictorial representation of entity classes, relationship classes, attribute classes, and their interconnections. DAG gives dependence among the attribute classes. Local distribution classes are probability distribution for dependence among attribute classes. Therefore, it is the graphical language for probabilistic entity relationship (PER) model and can also implement Plate model, where plate model is similar to PRM but suitable for statistician and PRM is used for computer users. It can perform probabilistic inference about attribute classes.

It can represent restricted relationships, self-relationships, partial relationship existence, and probabilistic relationships. It does not support first order formulas and quantifier, but due to dependence on random variable semantics it can be extended to FOL.

\section{BAYESIAN NETWORK WITH SEMANTIC WEB LANGUAGES}

This section mainly will go through the proposals based on an extension of RDF, an extension of $\operatorname{RDF}(\mathrm{S})$, and two proposals based on extension of OWL i.e. BayesOWL, OntoBayes, and MEBN and PR-OWL 2.0.

\subsection{Probabilistic extension of RDF}

Fukushige has proposed an implementation to integrate probability with RDF in the form of vocabulary [4]. Framework has been proposed on the basis of RDF and Bayesian network to calculate probability distribution. RDF is widely used language to represent ontology in semantic web and several successful software had been developed to create ontology using RDF and perform reasoning about that. This framework doesn't support any standard query language of semantic web instead Bayesian inference was the sole reasoning technique. The given framework starts by creating a vocabulary of propositions along with probability, which leads to a RDF graph. RDF graph should be converted into Bayesian network to perform reasoning.

An example of probabilistic relationship is borrowed from [6] for metastatic cancer. Vocabulary is created in N3 language [7], which includes propositions, negations, unconditional probabilities, conditional probabilities, observations and posteriors. This model can represent specific classes of problems with less expressiveness.

\subsection{Extension to $\mathrm{RDF}(\mathrm{S})$}

Holi and Hyvonen have used Bayesian network to compute degrees of overlap or ambiguous uncertainty between concepts of taxonomy [12]. $\operatorname{RDF}(\mathrm{S})$ is used to represent concept and partial subsumption. Concepts of taxonomies are represented as different sets in Venn diagram which helps to create overlap table for every concept. It uses a DAG to represent partial overlap where each node represents a concept which is annotated with mass i.e. size of the set. The graph is converted from partial overlap to a solid path structure using breadth first search algorithm. Overlap values will be interpreted as a conditional probabilities and efficient evidence propagation algorithm is used to calculate overlap computation. An individual will be matched with the query concept by computing the degrees of overlap, where degree of 
overlap plays significant role to measure the relevance of the concept in information retrieval techniques. BayesOWL can be seen as next methodologies on the basis of this approach.

\subsection{BayesOWL}

BayesOWL was one of the successful approaches to represent uncertain information based on probabilistic framework, which uses OWL to represent information in knowledge base [5]. It has been elaborated uncertain reasoning and mapping of ontology as two major applications of Bayesian Network.

This framework augments OWL with BN to represent uncertainty and perform uncertain reasoning. It gives a methodology to construct BN using OWL taxonomy for which five structural translation rules have been proposed. It is a mechanism to express OWL ontologies as Bayesian Network by adding additional nodes according to following constructors shown in Table 1.

On the basis of supported constructors, conversion of OWL taxonomy to a BN DAG is performed in two steps a) Structural Translation and b) Conditional Probability Table creation.

For structural translation, it uses two types of nodes a) Concept nodes b) L-nodes. Concept nodes are used for regular concept class. L-nodes are used for modeling relations among concept nodes where relations are logical operations and works as bridge between concept nodes. L-nodes will be created as leaf nodes to avoid cycle. It has two types of properties and can be classified as prior and conditional properties. Figure 4 shows an example of owl:unionOf constructor from [5]

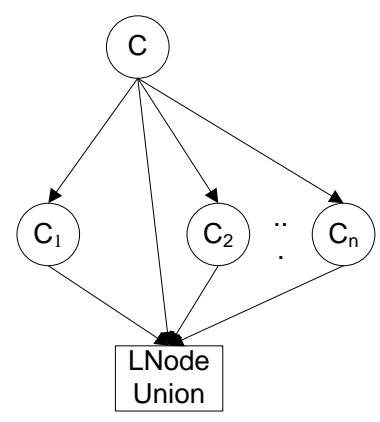

Figure 4 Conversion of union of concept nodes into LNodeUnion for Bayesian Network [5]

Here, Union of concept classes $C_{i}(i=1 \ldots n)$ is defined as $C$, using constructor "owl:unionOf" as partial BN. Conditional probability table will be created for both types nodes (Concept nodes as well as L-nodes) on the basis of proposed logical relations, which will include prior probability as well as conditional probability.

Piece-wise probability constraints can be used to construct CPTs with help of Decomposed-Iterative Proportional Fitting Procedure (D-IPFP) algorithm. Probabilistic inference plays major role for reasoning purposes in the proposed approach.
Three types of BN connections are allowed: serial connections, diverging connections and converging connections as shown in Figure 5.

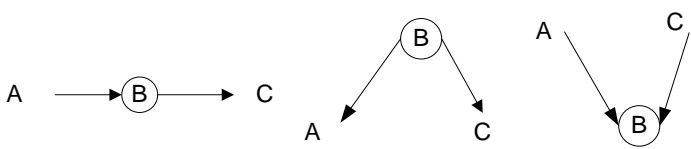

Figure 5 Bayesian Network connections a. Serial b. Diverging c. Converging [5]

BayesOWL supports only three basic reasoning within and across same ontology i.e. concept satisfiability, concept overlapping and concept subsumption, where concept satisfiablility checks whether a perfect description of a concept is available, concept overlapping decides the degree of overlap between a concept and its description, and at last concept subsumption finds whether a concept follows the properties of a given description.

The advantage of BayesOWL is that it reduces the cost and user efforts, since it doesn't enforce to modify OWL and ontologies. In addition to that it is not highly dependent on syntax instead of semantics as well as it can be used to translate either partial ontology or conditional probability table into $\mathrm{BN}$ according to user requirement in consistent fashion.

Limitations of BayesOWL: It cannot deal with multi-valued random variable. In translation of ontology, it does not consider the instances, the specific data type, and properties represented by BayesOWL.

\subsection{OntoBayes}

It is an ontology-driven Bayesian model for uncertain knowledge representation [25] [34]. OntoBayes is a decisiontheoretic design analysis. It integrates Bayesian network into web ontology language to annotate the ontology with Bayesian probability along with dependency relationship. OntoBayes deals only with discrete random variable but Boolean random variable as a special case. It does not deal with continuous random variable.

To provide probabilistic integration to OWL, only three classes have been introduced viz. PriorProb, CondProb and FullProbDist. PriorPrab and CondProb have only one property Probvalue and FullProbDist has two properties hasPrior and hasCond. PriorProb deals with unconditional probability and CondProb deals with conditional probability. FullProbDist deals with joint probability distribution table.

It uses two different graphical representation one for Bayesian graph and other for OWL. Bayesian graph depends on properties of OWL classes, and OWL graph depends on classes and properties. Although both graphs will have subject, predicate and object in the graph, but Bayesian graph will use only one predicate <rdfs:dependsOn>.

One major advantage of OntoBayes is that it can represent cyclic dependency and can deal with multi-valued random variable. For more expressivity, some approaches based on 
Table 1. Supported Constructor [5]

\begin{tabular}{|l|c|c|c|}
\hline Constructor & DL Notation & Class Axiom & Logical Operator \\
\hline rdfs:subClassOf & $\mathrm{C} 1 \sqsubseteq \mathrm{C} 2$ & $*$ & \\
\hline owl:equivalentClass & $\mathrm{C} 1 \equiv \mathrm{C} 2$ & $*$ & \\
\hline owl:disjointWith & $\mathrm{C} 1 \sqsubseteq \neg \mathrm{C} 2$ & $*$ & $*$ \\
\hline owl:unionOf & $\mathrm{C} 1 \sqcup \ldots \sqcup \mathrm{C} 2$ & & $*$ \\
\hline owl:intersectionOf & $\mathrm{C} 1 \sqcap \ldots \sqcap \mathrm{C} 2$ & & $*$ \\
\hline owl:complementOf & $\neg \mathrm{C}$ & & \\
\hline
\end{tabular}

\subsection{MEBN and PR-OWL 2.0}

Probabilistic Web Ontology Language (PR-OWL) [11] provides uncertainty representation using OWL constructs based on Multi-Entity Bayesian Network (MEBN) logic [9] and it is successfully implemented in UnBBayes [21] graphical user interface to model a probabilistic ontology [2], [8], [13], [14], [15], [18], [24], [26], [27] and [37]. MEBN logic is an extended form of Bayesian network along with expressive power of first order logic formula, where Bayesian network will encode a set of dependent evidences in graphical form and degree of belief of evidence can be interpreted with the help of constraints specified using first-order formulas. Basic constructs of MEBN are MTheory, MFrag, random variable, and entities.

MTheory is the label to combine multiple MFrags together and a probabilistic ontology must have at least one MTheory to represent the model. It will ensure the consistency of probabilistic ontology by checking unique joint probability distribution table. To perform the query, one has to provide domain specific entity instances i.e. knowledge base then Bayesian inference will be used for query and learning of new evidences.

MFrag is the combination of random variables, and fragment graph, where random variable will be represented as resident node along with conditional probability distribution table and fragment graph will show the dependence among random variables in the form of directed acyclic graph. A MFrag will give a template of a Bayesian network, which can be instantiated multiple times by binding its arguments to domain entity identifiers to create instances of its random variables. Resident node will work as input node to the other MFrag of ontology, where union of input node and resident node will be used as parent nodes in the fragment graph that is why the probability distribution table of the child node will have entries for the states of all its parent nodes. Here, mutual exclusive and exhaustive number of states of random variable will be represented using entities.
The advantage of MEBN over simple Bayesian network is that it can represent repeated structure of Bayesian network and it allows dynamic infinite number of instances of a Bayesian network structure. MEBN logic can represent various types of uncertainty like attribute value uncertainty, existence uncertainty, number uncertainty, referential uncertainty, structural uncertainty, and type uncertainty [9].

MEBN logic is successfully implemented using PR-OWL upper ontology, which is based on basic model as shown in figure 5. Here, ovals represent class and arc represents relationship between classes.

PR-OWL 1.0 has two shortcomings 1) it cannot provide mapping to properties of OWL and 2) although it provides concept of meta-entities for the definition of complex types, it does not have type compatibility with OWL. Therefore, PROWL 2.0 has been proposed to overcome these shortcomings to provide better modeling of domain information [18].

\section{PROPOSED MODELS USING PR- OWL FRAMEWORK}

Although PR-OWL is in initial phase of establishment and it has not been widely accepted by semantic web community [36], but some relevant proposed works discussed in this section will present importance and applicability of it.

\subsection{Service based situation awareness}

There are 3 levels of situation awareness presented in [34], 1) Perception, 2) Understanding, and 3) Projection, understanding type of situation awareness have been considered in [28] for service-oriented information technology environments. For situation assessment of status or health of IT services understanding (the second level) is more suitable than perception (the first level), because perception is more probable for inaccuracy and incomplete.

It has been proposed to develop a PR-OWL framework based ontology definition that supports automated reasoning with uncertain service-based situation awareness. 


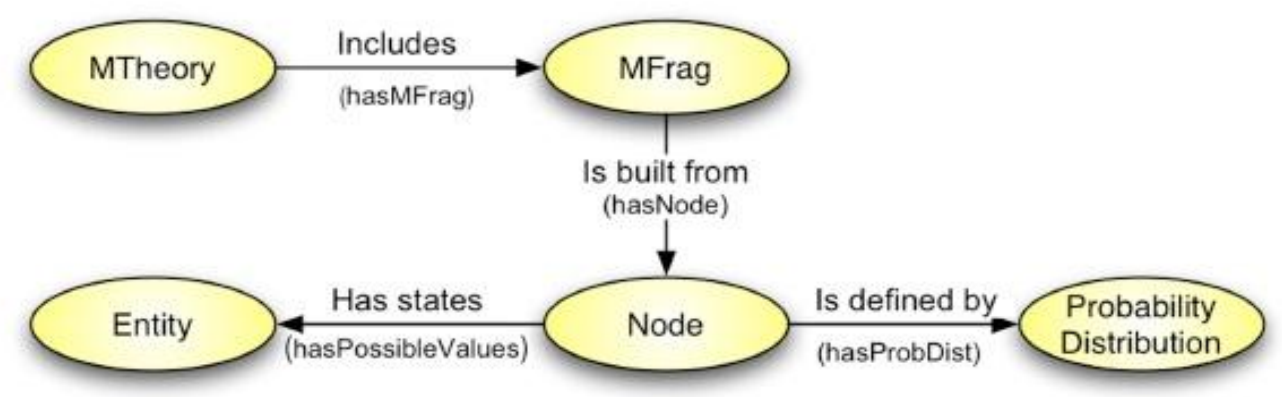

Figure 6 PR-OWL basic model [13]

\subsection{Research on Interactive Behaviour Analyzing in New-type Distributed Software System}

It has been tried to extract interactive behaviours produced by loosely-coupled software entities [33]. Behaviour of software entities is investigated on the basis of historical knowledge and current practical evidences. It has adopted the case based reasoning along with case reasoning.

It has been considered the new-type distributed software to supervise its group behaviour regarding behaviour of dynamic, accidental, correlative, and repeating properties. It has used MEBN to represent group behaviour as well as knowledge fusion of monitored evidence information and experimental knowledge. Fusion of problem space and solution space is useful to accurately analyze software behaviour credibility for current context.

\subsection{PROGONOS: Probabilistic Ontology for Net-centric operation systems}

It is Predictive Situation AWareness (PSAW) model to be used for U. S. Navy's FORCENet for prediction about battlefield, where huge amount of data is collected from sensors, human intelligence, and others [29]. It is modeled using PR-OWL framework, where it is based on two modules, the first module is used for performing reasoning and second module is used for simulation.

\subsection{Probabilistic Risk Assessment for Security Requirements: A Preliminary Study}

Risk assessment is the process of identifying risk factors and relationship among risk factors, which includes judgement as well as meta-judgement about the degree of certainty that they have in their judgements [35]. To represent the degree of certainty, it has been proposed a model, where security requirements and their causal relationships are represented using MEBN logic.

\subsection{A Model of an Ontology Oriented Threat Detection System (OOTDS)}

Object-oriented threat detection system is dynamic behaviour based system, which will be automatically upgraded according to changes in environment [14]. It is mainly based on two ontologies a) Threat detection ontology and b) Threat detection learning ontology, where the first one is used for threat detection and the second one is used for learning the dynamic changes in environment. Here, ontologies are created using PR-OWL model again.

\section{CONCLUSIONS}

Bayesian network can play a pivotal role in the representation of uncertainty and performing reasoning in semantic web. Survey on the Bayesian network for the representation of uncertainty has been discussed on the bases of description logic, object oriented, entity relational model approaches, as well as core works done so far for augmentation of Bayesian network with semantic web languages viz. $\mathrm{RDF}, \operatorname{RDF}(\mathrm{S})$, and OWL to represent uncertainty in knowledge bases, which provides efficient ways to perform reasoning to extract relevant information for specific purposes. The list of works done using PR-OWL has been discussed, which is a domain ontology based on MEBN logic and its relevance can been experienced by applying in various applications of real life.

\section{ACKNOWLEDGEMENTS}

We are very thankful to Dr. Rommel N. Carvalho and Dr. Paulo Cesar G. da Costa for giving permissions to use some contents and figures from their $\mathrm{Ph}$. D. theses and papers.

\section{REFERENCES}

[1] Stuart J. Russell \& Peter Norvig (2003) Artificial Intelligence - A Modern Approach, Second Edition, Pearson Education, Inc.

[2] Kumar Ravi \& G. Aghila (2013) Probabilistic Uncertainty Representation in Semantic Web, International Journal of Information and Computation Technology, Volume 3, Number 1 (2013), [SPECIAL ISSUE] pp. 27-33.

[3] Kenneth J. Laskey and K. B. Laskey, (2008) "Uncertainty reasoning for the world wide web: Report on the URW3-XG incubator group," W3C, URW3-XG, 2008. http://www.w3.org/2005/Incubator/urw3/XGRurw3-20080331/

[4] Yoshio Fukushige (2005) Representing Probabilistic Knowledge in the Semantic Web, available http://www.w3.org/2004/09/13Yoshio/PositionPaper.html

[5] Z. Ding (2005) BayesOWL: A Probabilistic Framework for Semantic Web. Doctoral dissertation. Computer Science and Electrical Engineering. 2005, University of 
Maryland, Baltimore County: Baltimore, MD, USA. p. 168

[6] J. Pearl (1998) Probabilistic reasoning in intelligent systems: networks of plausible inference, Morgan Kaufmann, 1988.

[7] An RDF language for the Semantic Web Available: http://www.w3.org/DesignIssues/Notation3.html

[8] Rommel Carvalho, Kathryn Laskey, Paulo Costa, Marcelo Ladeira, Laecio Santos and Shou Matsumoto (2010) UnBBayes: Modeling Uncertainty for Plausible Reasoning in the Semantic Web, Semantic Web, Gang Wu (Ed.), ISBN: 978-953-7619-54-1, InTech, Available from:

http://www.intechopen.com/books/semanticweb/unbbaye s-modeling-uncertainty-for-plausible-reasoning-in-thesemantic-web

[9] K. B. Laskey (2008) MEBN: A language for first-order Bayesian knowledge bases, Artificial Intelligence 172 (2008) 140-178

[10] F. Baader \& W. Nutt (2003) Basic Description Logics. Chapter in The Description Logics Handbook: Theory, Implementation and Applications. Baader, F.; Calvanese, D.; McGuiness, D.; Nardi, D.; Patel-Schneider, P.; editors. 1st edition, [47-100]. Cambridge, UK: Cambridge University Press

[11] P. C. G. Costa (2005) Bayesian Semantics for the Semantic Web, Ph. D. thesis; George Mason University.

[12] M. Holi, E. Hyvonen (2004) A method for modeling uncertainty in Semantic Web taxonomies, in: Proceedings WWW-2004, ACM Press, 2004, pp. 296297

[13] Rommel N. Carvalho, Kathryn B. Laskey, Paulo C. G. Costa, Marcelo Ladeira, Laécio L. Santos, and Shou Matsumoto (2013) Probabilistic Ontology and Knowledge Fusion for Procurement Fraud Detection in Brazil, F. Bobillo et al. (Eds.): URSW 2008-2010/UniDL 2010, LNAI 7123, pp. 19-40, 2013. Springer-Verlag Berlin Heidelberg.

[14] Karol Banczyk, Henryk Krawczyk (2008) A model of an Ontology Oriented Threat Detection System (OOTDS), Proceedings of the 2008 1st International Conference on Information Technology, IT 2008, 19-21 May 2008, Gdansk, Poland

[15] Daniel Vincen, Dafni Stampouli, Gavin Powell (2009) Foundations for System Implementation for a Centralised Intelligence Fusion Framework for Emergency Services, 12th International Conference on Information Fusion Seattle, WA, USA, July 6-9, 2009

[16] S. Tobies (2001) Complexity Results and Practical Algorithms for Logics in Knowledge Representation, PhD thesis, RWTH Aachen, Germany.

[17] I. Horrocks, U. Sattler (2005) A tableaux decision procedure for SHOIQ, in: Proceedings IJCAI-2005, 2005, pp. 448-453 (Extended version to appear in J. Autom. Reason.).

[18] Rommel N. Carvalho, Kathryn B. Laskey, and Paulo C.G. Costa (2013) PR-OWL 2.0 - Bridging the Gap to OWL Semantics, F. Bobillo et al. (Eds.): URSW 2008-
2010/UniDL 2010, LNAI 7123, pp. 1-18, 2013. Springer-Verlag Berlin Heidelberg.

[19] T. Lukasiewicz (2002) Probabilistic default reasoning with conditional constraints, Ann. Math. Artif. Intell. 34 (1-3)35-88.

[20] Ronan Daly, Qiang Shen and Stuart Aitken, (2011) Learning Bayesian networks: approaches and issues, The Knowledge Engineering Review, Vol. 26:2, 99-157. Cambridge University Press, 2011, doi: $10.1017 / \mathrm{S} 0269888910000251$

[21] Paulo C. G. COSTA, Kathryn B. Laskey, PR-OWL: A Framework for Probabilistic Ontologies "UnBBayes - the UnBBayes site," http://unbbayes.sourceforge.net/

[22] D. Koller, A. Y. Levy, and A. Pfeffer, (1997) PCLASSIC: A Tractable Probabilistic Description Logic. Paper presented at the Fourteenth National Conference on Artificial Intelligence (AAAI-97), July 27-31. Providence, RI, USA

[23] Pfeffer, A.; Koller, D.; Milch, B.; and Takusagawa, K. T. (1999) SPOOK: A System for Probabilistic ObjectOriented Knowledge Representation. In Proceedings of the Fifteenth Conference on Uncertainty in Artificial Intelligence, pp. 541-550, July 30 - August 1 . Stockholm, Sweden Sowa, J. F. (2000). Knowledge Representation: Logical, Philosophical, and Computational Foundations. Pacific Grove, CA, USA: Brooks/Cole.

[24] R. N. Carvalho (2008) Plausible reasoning in the semantic web using Multi-Entity bayesian networks MEBN, M.Sc., University of Braslia, Brasilia, Brazil, Feb. 2008.

[25] Y. Yi (2007) A framework for decision support systems adapted to uncertain knowledge. Ph.D. thesis; Fakultat fur Informatik der Universitat Fridericiana zu Karlsruhe.

[26] Rommel N. Carvalho (2011) Probabilistic Ontology: Representation and Modelling Methodology, Ph. D thesis; George Mason University, Brazil.

[27] Rommel N. Carvalho, Laécio L. Santos, Marcelo Ladeira, Paulo C. G. Costa (2007) A GUI Tool for Plausible Reasoning in the Semantic Web using MEBN. In Proceedings of the Seventh International Conference on Intelligent Systems Design and Applications (ISDA 2007). Mourele, L.; Nedjah, N.; Kacprzyk, J.; and Abraham, A. (eds.); pp. 381-386. October 22-24, 2007, Rio de Janeiro, Brazil.

[28] Stephen. C. Dinkel, William Hafner, Paulo Costa Sumitra Mukherjee (2011) Uncertainty Reasoning for Service-based Situational Awareness Information on the Semantic Web, 2011 IEEE International MultiDisciplinary Conference on Cognitive Methods in Situation Awareness and Decision Support (CogSIMA), Miami Beach, FL

[29] R. N. Carvalho, P. C. G. Costa, K. B. Laskey, and K. Chang (2010) "PROGNOS: predictive situational awareness with probabilistic ontologies," in Proceedings of the 13th International Conference on Information Fusion, Edinburgh, UK, Jul. 2010.

[30] Lise Getoor, Nir Friedman, Daphne Koller, Avi Pfeffer and Ben Taskar, Probabilistic Relational Models 
[31] David Heckerman, Christopher Meek, and Daphne Koller (2004) Probabilistic Models for Relational Data, March 2004, Microsoft Research and Stanford University, Technical Report, MSR-TR-2004-30

[32] R. N. Carvalho, R. Haberlin, P. C. G. Costa, K. B. Laskey, and K. Chang (2011) "Modeling a probabilistic ontology for maritime domain awareness," in Proceedings of the 14th International Conference on Information Fusion, Chicago, USA, Jul. 2011.

[33] Changyun Li, Junfeng Man, Zhibing Wang, and Xiangbing Wen (2010) Research on Interactive Behavior Analyzing in New-type Distributed Software System, Proceedings of 2010 Conference on Dependable Computing (CDC'2010) November 20-22, 2010, Yichang, China

[34] M. R. Endsley (1995) Toward a Theory of Situation Awareness in Dynamic Systems, Human Factors: The Journal of the Human Factors and Ergonomics Society, vol. 37, pp. 32-64, 1995.

[35] Seok-Won Lee (2011) Probabilistic Risk Assessment for Security Requirements: A Preliminary Study, 2011 Fifth International Conference on Secure Software Integration and Reliability Improvement, IEEE computer society, DOI 10.1109/SSIRI.2011.12

[36] Amandine Bellenger, Sylvain Gatepaille (2008) Uncertainty in Ontologies: Dempster-Shafer Theory for Data Fusion Applications, 2008.

[37] Kumar Ravi, Sheopujan Singh (2013) Risk Prediction for Production of an Enterprise, International Journal of Computer Applications Technology and Research (IJCATR), Volume 2 Issue 3 May-June 2013, doi: 10.7753/IJCATR0203.1006 